\title{
Forschungsnotiz
}

Barbara Dewein, Stefan Engelberg, Susanne Hackmack, Wolfram Karg, Birte Kellermeier-Rehbein, Peter Muhlhäusler, Daniel Schmidt-Brucken, Christina Schneemann, Doris Stolberg, Thomas Stolz, Ingo H. Warnke

\section{Forschungsgruppe Koloniallinguistik: Profil - Programmatik - Projekte}

\footnotetext{
Prof. Dr. Stefan Engelberg: Institut für Deutsche Sprache (IDS), Leiter der Abteilung Lexik, Postfach 1016 21, D-68016 Mannheim, E-Mail: engelberg@ids-mannheim.de Thomas Stolz: FB 10: Linguistik, Universität Bremen, Postfach 330 440, D-28334 Bremen, E-Mail: stolz@uni-bremen.de

Prof. Dr. Ingo H. Warnke: FB 10: Deutsche Sprachwissenschaft, Universität Bremen, Postfach 330 440, D-28334 Bremen, E-Mail: iwarnke@uni-bremen.de

1 Überblick, Ziele und begriffliche Einordnung der Forschungsgruppe Koloniallinguistik

2 Arbeitsfelder, Forschungsmethodik und Quellen

2.1 Forschungsfeld Sprachkontakt und Sprachwandel

2.2 Forschungsfeld Historiographie der Linguistik

2.3 Forschungsfeld Diskurslinguistik

2.4 Forschungsfeld Sprach- und Sprachenpolitik

2.5 Quellen

3 Die Forschungsgruppe Koloniallinguistik im linguistischen Kontext und in der Lehre Danksagung

Literatur
}

\section{1 Überblick, Ziele und begriffliche Einordnung der Forschungsgruppe Koloniallinguistik}

Koloniallinguistik ist ein neues, interdisziplinäres sprachwissenschaftliches Forschungsprogramm, das sich erstmalig der systematischen Erfassung, Ordnung und Deutung aller linguistisch relevanten Phänomene widmet, die in Zusammenhang mit Kolonialismus stehen. Untersucht werden dabei die im kolonialen Kontext $\mathrm{zu}$ beobachtenden Sprachkontakt- und Sprachwandelphänomene, die zur Kolonialzeit und darüber hinaus betriebene Grammatikographie und Lexikographie, die wir als kolonialzeitliche Sprachforschung bezeichnen, die historischen Diskurse über und in Kolonien sowie die Sprach(en)politik im kolonialen Kontext. 
Ein Forschungsdesiderat ergibt sich dabei einerseits aus bislang unerschlossenen, sprachwissenschaftlich relevanten Daten in Archiven und Bibliotheken, und andererseits aus dem Umstand, dass entsprechende Forschungen bisher Marginalien der Sprachgeschichtsschreibung geblieben sind. Die Forschungsgruppe Koloniallinguistik - zunächst getragen von Forschungsinstitutionen in Bremen, Mannheim und Wuppertal - greift dieses Desiderat auf. Bei aller Breite der relevanten Forschungsfelder sind die Mitglieder verbunden durch die Überzeugung, dass die beobachteten linguistischen Phänomene nur unter Bezugnahme auf die spezifischen historischen Machtkonstellationen des Kolonialismus erklärt werden können, durch die Bemühung zur Erschließung historischer objekt- und metasprachlicher Quellen, durch das Bestreben, einen Beitrag zur kritischen Analyse der kolonialen Vergangenheit zu leisten, und durch den Wunsch, interdisziplinär und international zu arbeiten, sowie insbesondere mit Wissenschaftlern und Wissenschaftlerinnen aus ehemaligen Kolonialgebieten zu kooperieren.

Ihre begriffliche Grundlage sieht die Forschungsgruppe Koloniallinguistik in Kolonialismus als einem direkten und vermittelten Kontakt von ethnisch bzw. national definierten Gruppen in einer sozial asymmetrischen Machtkonstellation, in der eine hegemoniale Gruppe aus geographischer oder anderer Distanz heraus Herrschaft über die anderen beteiligten Gruppen ausübt oder auszuüben sucht und dabei eigene politische, wirtschaftliche, kulturelle etc. Interessen dominant verfolgt. In einem engeren, einschlägigen Sinne sei unter Kolonialismus der Prototyp einer europäisch geprägten Überseeexpansion von Staaten unter Inbesitznahme von Territorien zwecks wirtschaftlicher Ausbeutung, Erhöhung des nationalen Prestiges, militärischer Kontrolle, Schaffung von Siedlungsgebiet etc. verstanden. Unser Interesse richtet sich dabei vor allem auf den deutschen Kolonialismus.

Den koloniallinguistischen Forschungsansätzen nützlich ist eine begriffliche Differenzierung der Ausdrücke kolonial (räumlich-zeitlicher Bezug auf die Kolonien etwa des Deutschen Reiches von 1884 bis 1919), kolonisatorisch (koloniale Praxis mit machtpolitischem, wirtschaftlichem, militärischem, kulturellem etc. Anspruch) und kolonialistisch (ideologische Haltung, die aber nicht an die historische Periode des faktischen Kolonialismus gebunden sein muss, sondern zeitlich auch vor- und nachgelagert sein kann). Weiterhin begrifflich abzugrenzen ist das rezente Forschungsprogramm der Koloniallinguistik von kolonialzeitlicher Sprachforschung im Sinne grammatiko- und lexikographischer Beschreibungen und Kodifikationen ehemals kolonialer Sprachen während der Kolonialzeit.

Die Bezeichnung Koloniallinguistik selbst ist nicht unproblematisch, da eine Übernahme der Bedeutung von kolonial ein simplifizierendes und vielleicht sogar revisionistisches Geschichtsbild suggerieren könnte. Auf diese Problematik wollen wir ausdrücklich verweisen, zumal eine kritische Sprachreflexion in den letzten Jahren immer wieder eingefordert wurde (vgl. Arndt/Hornscheidt 2009; Nduka- 
Agwu/Hornscheidt 2010; Arndt/Ofuatey-Alazard 2011). Wir verstehen unter Koloniallinguistik nicht ein kolonialaffirmatives Forschungsprogramm, sondern eines, das im Gegenteil koloniale Machtkonstellationen in ihrer Verschränkung mit sprachlichen Gegenständen einer datengestützten, kritischen Analyse unterzieht. Jegliche revisionistische Haltung lehnen wir ab und liegt unseren Forschungsinteressen fern.

Die gegenwärtigen Aktivitäten der Forschungsgruppe Koloniallinguistik umfassen u. a. die Herausgabe der Publikationsreihe „Koloniale und Postkoloniale Linguistik/Colonial and Post-Colonial Linguistics“ (Berlin: Akademie-Verlag), die Veranstaltung regelmäßiger Tagungen und Workshops, die zum Teil gemeinsame Vorbereitung und Durchführung von Drittmittelprojekten sowie die Information der Fachöffentlichkeit (www.fb10.uni-bremen.de/fkl). Eine Vertiefung der hier vorgestellten Gesichtspunkte und jeweils ein ausführlicher Literaturbericht und ideengeschichtlicher Rückblick finden sich in Warnke (2009a) und Stolz/Vossmann/Dewein (2011a).

\section{Arbeitsfelder, Forschungsmethodik und Quellen}

\subsection{Forschungsfeld Sprachkontakt und Sprachwandel}

Die koloniallinguistische Sprachkontakt- und Sprachwandelforschung befasst sich mit dem Entstehen, dem Wandel und dem Verschwinden von Sprachen unter kolonialen Herrschaftssystemen. Dazu gehören unter anderem Forschungen zur Entwicklung von Misch-, Pidgin- und Kreolsprachen, zu in den indigenen Sprachen hervorgerufenen lexikalischen und grammatischen Veränderungen, zur Entwicklung von Sprachinseln etc. Es wird auf Methoden der Lehnwortforschung und Kontaktlinguistik zurückgegriffen. (Vgl. zu diesem Forschungsfeld Engelberg 2006a, 2006b, 2010, 2012; Engelberg/Scholz/Stolberg 2012; Stolberg 2011, 2012; Weber in Vorb.)

\subsection{Forschungsfeld Historiographie der Linguistik}

Die koloniallinguistische Historiographie befasst sich mit der kolonialzeitlichen Sprachforschung, so wie sie von Missionierenden, Philologinnen und Philologen, Angestellten der Kolonialverwaltung, Forschungsreisenden und anderen betrieben wurde. Konkret beschäftigt sie sich unter anderem mit der Analyse und Edition kolonialzeitlicher Grammatiken und Wörterbücher, mit kolonialzeitlichen linguistischen Theorien und Kategoriensystemen sowie mit der Geschichte der 
Feldforschungsmethodik, -methodologie und -ethik. Ein Ziel dieses Forschungsfeldes ist es auch, den ehemals beforschten Sprachgemeinschaften kolonialzeitliche Publikationen in Editionen zugänglich zu machen. In methodischer Hinsicht werden dabei personen- und wissenschaftsgeschichtliche Verfahren angewandt, sowie ein wissenschaftssoziologisches Interesse mit historischer Ausrichtung verfolgt. (Vgl. zu diesem Forschungsfeld Stolz 2007, 2011a, b, 2012; Stolz/Schneemann/Dewein/Chung 2011; Vossmann 2011; Dewein 2011.)

\subsection{Forschungsfeld Diskurslinguistik}

Die Erforschung des Kolonialdiskurses im Sinne der Gesamtheit von Äußerungen über und in Kolonien von Kolonisierenden und Kolonisierten beinhaltet unter anderem Forschungsarbeiten zu den sprachlichen Mitteln der Konstituierung und Thematisierung kolonialer Sachverhalte, zur diskursiven Konstruktion von Konzepten unter kolonial geprägten Bedingungen sowie zur sprachlichen Selbstpositionierung von Kolonisierten und Kolonisierenden. Dazu werden diskurslinguistische Verfahren der Korpus- und Einzeltextanalyse angewendet, die sich auf unterschiedliche sprachliche Phänomene wie lexikalische Muster und grammatische Konstruktionen beziehen, um Aufschluss über kolonialzeitliche Wissensbestände zu gewinnen. (Vgl. zu diesem Forschungsfeld Warnke 2009a, sowie die Beiträge in Warnke 2009b; Spitzmüller/Warnke 2011; Warnke/Schmidt-Brücken 2011, 2012.)

\subsection{Forschungsfeld Sprach- und Sprachenpolitik}

Die koloniallinguistische sprach- und sprachenpolitische Forschung befasst sich mit der gezielten Einflussnahme auf die Entwicklung einzelner Sprachen (Sprachpolitik) und auf die Sprachenverhältnisse (Sprachenpolitik). Dabei werden unter anderem Sprachenpolitik von Regierungen, Missionen und indigener Führung, der Umgang mit Sprachenvielfalt sowie Alphabetisierungsaktivitäten untersucht. Zugänge zur Sprach(en)politik findet die Forschung in historischen Quellen, da die herkömmlichen Verfahren der Sprach(en)politikforschung meist gegenwartsbezogen sind. (Vgl. zu diesem Forschungsfeld Mühlhäusler 2001; Engelberg 2008; Engelberg/Scholz/Stolberg 2012.) 


\subsection{Quellen}

Aufgrund der spezifischen Eigenschaften ihres Forschungsgegenstandes sieht sich die Forschungsgruppe Koloniallinguistik mit besonderen empirischen Problemen hinsichtlich der historischen textuellen Grundlagen ihrer Forschung und der Entwicklung geeigneter empirischer Methoden zur Datengewinnung konfrontiert. Alle vier Forschungsfelder der Koloniallinguistik arbeiten meist auf der Basis historischer, geschriebener Texte, die meist nur sehr schwer zu erschließen sind. Das liegt zum einen an teilweise sehr geringen Auflagen von relevanten Quellen, die etwa im Eigenverlag der Missionen vor Ort erschienen sind. Zum anderen findet sich ein großer Teil der relevanten Dokumente in nicht immer leicht zugänglichen Archiven. Die Erschließung der für die Koloniallinguistik relevanten textuellen Grundlagen ist daher eine wichtige Aufgabe. Sie beinhaltet die Sichtung und Erschließung unpublizierter Archivalien, die bibliographische Erfassung publizierten Materials, die Digitalisierung von Textbeständen und die Archivierung der (vermutlich nur) wenigen Tondokumente gesprochener Sprache.

\section{Die Forschungsgruppe Koloniallinguistik im linguistischen Kontext und in der Lehre}

Ein wichtiger Aspekt, der die Forschungsrelevanz der Koloniallinguistik deutlich hervortreten lässt, ist die enge Verzahnung von sprachwissenschaftlichen Forschungsfeldern, die üblicherweise wenig Notiz voneinander nehmen. Neben der innerlinguistischen Zusammenarbeit, die durch den Zusammenhang innerhalb der Forschungsgruppe Koloniallinguistik gegeben ist, wird die Interdisziplinarität durch Kooperation mit Geschichtswissenschaften, Afrikanistik, Austronesistik und Postkolonialen Studien erweitert. Mit der Missionarslinguistik (vgl. Hovdhaugen 1996; Zimmermann 2004) teilt die Koloniallinguistik das Interesse an Fragestellungen der linguistischen Fachgeschichte, d. h. dass in beiden Forschungsprogrammen die Leistung der deskriptiv-linguistisch tätigen kolonialzeitlichen Sprachforschenden einer kritischen Würdigung unterzogen wird. Die Abgrenzung findet sich dagegen im räumlichen, zeitlichen und sprachlichen Bezug beider Forschungsfelder, der für die Forschungsgruppe Koloniallinguistik zunächst beim deutschen Kolonialismus liegt. Mit den Postkolonialen Studien findet die Koloniallinguistik ihre Schnittmenge hauptsächlich im Forschungsfeld Diskurslinguistik, die unter anderem an diskursanalytischer Ideologieforschung bezüglich der kolonialzeitlichen Sprachforschung interessiert ist (vgl. Errington 2001, 2008). 
Über die Forschung hinaus, doch eng auf sie bezogen, können die Erkenntnisse der Forschungsgruppe Koloniallinguistik schließlich auch dem wissenschaftlichen Nachwuchs und zukünftigen Lehrenden in universitären Lehrveranstaltungen erschlossen werden (vgl. Kellermeier-Rehbein 2012). Indem die Lehre neue Erkenntnisse thematisiert, lässt sie die Forschung durch kritische Diskussionen und Anregungen davon profitieren. Die Koloniallinguistik bietet eine Fülle von Themen und Unterrichtsgegenständen für universitäre Lehrveranstaltungen, die sich aus ihren oben skizzierten Arbeitsfeldern und Zielsetzungen ergeben. Sie können didaktisch so vermittelt werden, dass sie aus rein sprachwissenschaftlicher Perspektive oder interdisziplinär unter Zuhilfenahme weiterer Fachwissenschaften erarbeitet werden.

Danksagung: Ausdrücklicher Dank für kritische Anmerkungen und Diskussionen, die zur Konturierung des Programms der Forschungsgruppe Koloniallinguistik wesentlich beigetragen haben, geht an Klaus Zimmermann (Bremen), Brigitte Weber (Klagenfurt), Beatrix Busse (Heidelberg) und alle Mitarbeiterinnen und Mitarbeiter, die sich aktiv in die Diskussion eingebracht haben.

\section{Literatur}

Arndt, Susan/Hornscheidt, Antje (Hrsg.) (2009): Afrika und die deutsche Sprache. Ein kritisches Nachschlagewerk. 2. Aufl. Münster: Unrast.

Arndt, Susan/Ofuatey-Alazard, Nadja (Hrsg.) (2011): Wie Rassismus aus Wörtern spricht. (K)Erben des Kolonialismus im Wissensarchiv deutsche Sprache. Ein kritisches Nachschlagewerk. Münster: Unrast.

Dewein, Barbara (2011): Reduplikation als Thema in Hermann Costenobles Die Chamoro Sprache. In: Stolz/Vossmann/Dewein (2011b), 249-262.

Engelberg, Stefan (2006a): The Influence of German on the Lexicon of Palauan and Kosraean. In: Allen, Keith (Hrsg.): Selected Papers from the 2005 Conference of the Australian Linguistic Society. Online unter: http://www.als.asn.au/proceedings/als2005.html (5.12.2011).

Engelberg, Stefan (2006b): Kaisa, Kumi, Karmoból. Deutsche Lehnwörter in den Sprachen des Südpazifiks. In: Sprachreport 22 (4), 2-9.

Engelberg, Stefan (2008): The German Language in the South Seas: Language Contact and the Influence of Language Politics and Language Attitudes. In: Schulze, Mathias et al. (Hrsg.): German Diasporic Experiences: Identity, Migration, and Loss. Waterloo, ON: Wilfrid Laurier University Press, 317-329.

Engelberg, Stefan (2010): An inverted loanword dictionary of German loanwords in the languages of the South Pacific. In: Dykstra, Anne/Schoonheim, Tanneke (Hrsg.): Proceedings of the XIV EURALEX International Congress (Leeuwarden, 6-10 July 2010). Ljouwert (Leeuwarden): Fryske Akademy, 639-647. 
Engelberg, Stefan (2012): Historische Sprachkontaktforschung zur deutschen Sprache im Südpazifik - Ansatz zu einer Quellen- und Dokumentenkunde der Deutschen Koloniallinguistik. Erscheint in: Engelberg/Stolberg (2012).

Engelberg, Stefan/Scholz, Ineke/Stolberg, Doris (2012): Interaktionszentren des Sprachkontakts in Deutsch-Neuguinea: ein sprachkartographisches Projekt. Erscheint in: Engelberg/Stolberg (2012).

Engelberg, Stefan/Stolberg, Doris (Hrsg.) (2012): Sprachwissenschaft und kolonialzeitlicher Sprachkontakt. Sprachliche Begegnungen und Auseinandersetzungen. Berlin: AkademieVerlag (= Koloniale und Postkoloniale Linguistik 3).

Errington, Joseph (2001): Colonial Linguistics. In: Annual Review of Anthropology 30, 19-30.

Errington, Joseph (2008): Linguistics in a Colonial World. A Story of Language, Meaning, and Power. Oxford: Blackwell.

Hovdhaugen, Even (1996): Missionary grammars: an attempt at defining a field of research. In: Hovdhaugen, Even (eds.): ... and the word was God: missionary linguistics and missionary grammar. Münster: Nodus, 9-22.

Kellermeier-Rehbein, Birte (2012): Koloniallinguistik aus hochschuldidaktischer Perspektive. Erscheint in: Engelberg/Stolberg (2012).

Mühlhäusler, Peter (2001): Die deutsche Sprache im Pazifik. In: Hiery, Hermann Joseph (Hrsg.): Die deutsche Südsee 1884-1914. Ein Handbuch. Paderborn et al.: Schöningh, 239-262.

Nduka-Agwu, Adibeli/Hornscheidt, Antje (Hrsg.) (2010): Rassismus auf gut Deutsch. Ein kritisches Nachschlagewerk zu rassistischen Sprachhandlungen. Frankfurt am Main: Brandes \& Apsel.

Spitzmüller, Jürgen/Warnke, Ingo H. (2011): Diskurslinguistik. Eine Einführung in Theorien und Methoden der transtextuellen Sprachanalyse. Berlin/Boston: de Gruyter.

Stolberg, Doris (2011): Sprachkontakt und Konfession. Lexikalische Sprachkontaktphänomene Deutsch-Nauruisch bei den Missionaren Delaporte und Kayser. In: Stolz/Vossmann/Dewein (2011b), 285-304.

Stolberg, Doris (2012): Sprachkontakt in der Schule: Deutschunterricht in Mikronesien. Erscheint in: Engelberg/Stolberg (2012).

Stolz, Thomas (2007): The Kurze Geschichte der Marianen by Georg Fritz. A commented reedition. In: Schrader-Kniffki, Martina/Morgenthaler Garcia, Laura (Hrsg.): La Romania en interacción: entre historia, contacto y política. Ensayos en homenaje a Klaus Zimmermann. Frankfurt: Klaus Vervuert, 307-349.

Stolz, Thomas (2011a): Koloniallinguistischer Konkurrenzkampf auf den Marianen: über Grammatik und Wörterbücher der Chamorrosprache im frühen 20. Jahrhundert. In: Stolz/Vossmann/Dewein (2011b), 203-230.

Stolz, Thomas (2011b): German and Dutch contributions to Chamorro studies (1800-1920). In: Reid/Ridruejo/Stolz (2011), 201-226.

Stolz, Thomas (2012). Über die Wortmacherei, oder: Die Verschiebung der Wortgrenzen in der kolonialzeitlichen Sprachforschung (am Beispiel des Chamorro). Erscheint in: Engelberg/ Stolberg (2012).

Stolz, Thomas/Schneemann, Christina/Dewein, Barbara/Chung, Sandra (2011): The mysterious H. Who was the author of Die Chamoro Sprache? In: Stolz/Reid/Ridruejo (2011), 227-242.

Stolz, Thomas/Vossmann, Christina/Dewein, Barbara (2011a): Kolonialzeitliche Sprachforschung und das Forschungsprogramm der Koloniallinguistik: eine kurze Einführung. In: Stolz/Vossmann/Dewein (2011b), 7-30. 
Stolz, Thomas/Vossmann, Christina/Dewein, Barbara (Hrsg.) (2011b): Kolonialzeitliche Sprachforschung. Die Beschreibung afrikanischer und ozeanischer Sprachen zur Zeit der deutschen Kolonialherrschaft. Berlin: Akademie Verlag (= Koloniale und Postkoloniale Linguistik 1).

Vossmann, Christina (2011): Gertrude Hornbostels Aufzeichnungen im Lichte zweier Klassiker der Chamorroforschung. In: Stolz/Vossmann/Dewein (2011b), 231-248.

Warnke, Ingo H. (2009a): Deutsche Sprache und Kolonialismus. Umrisse eines Forschungsfeldes. In: Warnke (2009b), 3-62.

Warnke, Ingo H. (Hrsg.) (2009b): Deutsche Sprache und Kolonialismus. Aspekte der nationalen Kommunikation 1884-1919. Berlin/New York: de Gruyter.

Warnke, Ingo H./Schmidt-Brücken, Daniel (2011): Koloniale Grammatiken und ihre Beispiele Linguistischer Sprachgebrauch als Ausdruck von Gewissheiten. In: Stolz/Vossmann/Dewein (2011b), 31-54.

Warnke, Ingo H./Schmidt-Brücken, Daniel (2012): Was zählt im Kolonialdiskurs? Numeralia und Numeralität in kolonialen Grammatiken. Erscheint in: Engelberg/Stolberg (2012).

Weber, Brigitte (in Vorb.): Place names in Cameroon - a preliminary study.

Zimmermann, Klaus (2004): La construcción del objeto de la historiografía de la lingüística misionera. In: Zwartjes, Otto/Hovdhaugen, Evene (eds.): Missionary Linguistics/Lingüística misionera. Selected Papers from the First International Conference on Missionary Linguistics, Oslo, 13-16 March 2003. Amsterdam: Benjamins, 7-32. 